\title{
What is Different About Pulsed Field Ablation ... Everything?
}

\author{
David Haines ${ }^{1}$ \\ ${ }^{1}$ Beaumont Health System
}

January 5, 2022

What is Different About Pulsed Field Ablation ... Everything?

David E. Haines, MD

Beaumont Health System, Oakland University William Beaumont School of Medicine, Royal Oak, Michigan, USA

Funding: None

Disclosures: Dr. Haines is a consultant for Ablacon, Inc., Affera, Inc., Biosense Webster, Inc., Boston Scientific Corporation, Inc., Medtronic, Inc., and Philips Healthcare, Inc. He has equity interest in Ablacon, Inc. and Affera, Inc.

Corresponding author:

David E. Haines, MD Director, Heart Rhythm Center Professor, Cardiovascular Medicine Oakland University William Beaumont School of Medicine 3601 West 13 Mile Road Royal Oak, MI 48073 248-898-4176 dhaines@beaumont.edu

From the time of early preclinical reports of the efficacy, speed and safety of pulsed field ablation (PFA), the interventional electrophysiology community has been waiting in anxious anticipation for its clinical approval and release. As most people actively engaged in interventional electrophysiology know, PFA is the technology that creates myocardial lesions with trains of very high voltage pulses that are nanoseconds or microseconds in duration. ${ }^{1}$ This form of ablation is nonthermal, and cell injury/death is created by electroporation of the organelles and sarcolemmal membrane, with cell death occurring via apoptosis as well as other mechanisms. ${ }^{2}$ Because myocardial ablation is the result of an electrical field effect, electrode-tissue proximity is important but contact force is not. The physiological effect of acute PFA is striking in that there is immediate loss of electrical signal so that acute pulmonary vein isolation is achieved from a single shot catheter in under one second (figure 1). Preclinical animal studies have demonstrated effective chronic lesions that have been reliably transmural for atrial ablations. ${ }^{3}$ But the most compelling aspect of PFA is the apparent tissue selectivity of this energy form. At doses sufficient to create reliable transmural lesions in animals, there is little or no discernable injury to the esophagus, phrenic nerves, coronary arteries or distal pulmonary veins, in contrast to ablation with thermal techniques such as radiofrequency or cryoballoon ablation, where thermal collateral injury to contiguous structures can lead to substantial morbidity and death. ${ }^{4,5}$

So, what is the catch? Is there any downside to PFA compared to other ablation technologies? The answer is that we do not know. Despite a substantial body of preclinical experience published to date, clinical outcomes with PFA are just starting to be reported. In this issue of theJournal, Neven et al describe the acute pathological effects of PFA in a swine model over the first 60 minutes and compare them to findings from chronic animals at 3 weeks and 3 months. ${ }^{6}$ For their study, they mostly employed a suction electrode for epicardial delivery (some lesions created with a circular catheter under blood) and delivered a single unipolar cathodal pulse of mostly $200 \mathrm{~J}$ (some dose ranging performed with chronic lesions between 30 - 
$200 \mathrm{~J})$ at various sites on the right and left ventricles. Pathology on acute specimens was examined 0 to 60 minutes post ablation, and chronic specimens were examined after 3 weeks and 3 months. They reported that acute lesions showed contraction band necrosis and interstitial edema, and at $>/=3$ weeks, there was sharply demarcated connective tissue that evolved into scar.

What new insights have we gained from the present study that we can now apply to the burgeoning field of clinical PFA? Unfortunately, despite considerable effort put forth by these investigators, their results are not generalizable to the wider field of PFA. No study reporting outcomes using one proprietary PFA system will be necessarily applicable to results from a competing proprietary system. The field of catheter ablation has become accustomed to interchangeability of one RF generator or catheter for another. That is because the thermodynamics of electrical resistive heating of tissue is predictable, and there are limited operator parameters (power, time, contact force) and design parameters (electrode size, convective cooling) that affect tissue heating. The biological effects of hyperthermia are straightforward and reproducible; thus, it is possible for scientists and clinicians to compare experiences from one proprietary RFA system to another and draw some generalizable conclusions. ${ }^{7}$ Not so with PFA. To paraphrase an old saying, "When you've seen one PFA system, you've seenone PFA system." The operator-controlled parameters are familiar (energy level, catheter-tissue position), but the design parameter variations are dizzying and most importantly unknown to us. A precedent was set early in the development of this field that the PFA waveform was proprietary, and that the commercialized product was a "black box". A few of the parameters that can be modulated by the manufacturer include pulse amplitude, pulse duration, unipolar/bipolar, monophasic/biphasic, interpulse delay, interphase delay, pulse train number, pulse train duration and more. For example, the waveform used by Neven et al. was a milliseconds duration, monophasic damped sine wave generated by an external defibrillator (Lifepak 9, Physio-Control, Inc; Redmond, WA) and delivered in a unipolar cathodal fashion to an indifferent dispersive skin electrode. In contrast, most PFA systems deliver pulses are nanoseconds to microseconds in duration with a range of variation of the other parameters listed above (figure 2). Thus, the outcomes from ablation with a proprietary PFA system cannot and should not be extrapolated to different proprietary systems that may have dramatically different waveforms that result in different procedural outcomes.

One of the major unanswered questions in the field of PFA is what is the time course of clinical effect after energy delivery? The hope and anticipation has been that lesions created by PFA will be more durable than those created with thermal ablation techniques (RFA or cryoablation). A dramatic features of catheter ablation with PFA is that disappearance of the local electrograms is seen immediately after pulse delivery (figure 1). Unfortunately, this does not necessarily indicate that a permanent lesion has been created. Beyond the border of irreversible electroporation there is a penumbra of reversible electroporation manifested by loss of the electrical signal but maintenance of viability. In the heart, this has been described pathologically as a rim of tissue with enhanced with hematoxylin and eosin staining, and maintenance of mitochondrial activity as delineated by cytochrome $\mathrm{C}$ oxidase staining. ${ }^{8}$ Thus, the acute end point of conduction block and loss of local electrogram signal employed in RF ablation does not indicate that a permanent lesion has been made with PFA. With myocardial ablation, the extent of reversible electroporation beyond the border of permanent injury has not been fully characterized, nor has the time course of recovery of normal function. There are many anecdotal reports of pulmonary vein reconnection after acute PVI with PFA in patients with atrial fibrillation. Unfortunately, the present study did not report mapping data and compare the extent of acute physiological effect to the acute pathological lesions.

Therefore, a challenge for operators performing PFA is that acute electrogram voltage mapping cannot be used as a metric to assess therapeutic effect. The strategy adopted for PVI with PFA is to deliver a very large ablation dose, since injury to collateral structures is rare. ${ }^{9}$ The larger the energy delivery, the more likely it will be that permanent block is achieved. One experienced operator concluded "Go big or go home". Once again, the pulse wave morphology, pulse width and pulse train number are proprietary features of the system that are preselected. The operator can choose different pulse amplitudes, but in almost all cases the maximum setting is selected. Is there such a thing as too much energy delivery with PFA? In preclinical studies, very high energy delivery has been associated with large volume microbubble production 
and evidence of thermal effects (charring) at the electrode-tissue interface. Creating a large uncontrolled lesion may be appropriate for a narrowly defined anatomical target like the pulmonary vein antrum but may not be appropriate for targeting other arrhythmogenic substrates because of concern about excessive injury to the surrounding myocardium. The consequences of very high PFA energy delivery in the clinical setting are unknown but most experts believe that there should be an upper safe limit established for each unique PFA system. So, the manufacturers will decide the dosing that the clinician will use. Will the recommended dose be safe and effective for all patients going forward? Time will tell. Ideally, we should have a method to assess myocardial viability after PFA prior to removing catheters from the heart, so that touch up ablations with improved catheter-tissue proximity can be performed. With RFA, promising technologies like real time magnetic resonance imaging, ${ }^{10}$ near field ultrasound imaging ${ }^{11}$ and $\mathrm{NADH}$ fluorescence ${ }^{12}$ have been explored, but their utility in assessing acute PFA lesions is unknown.

The era of PFA is exciting and full of promise, but there are many unanswered questions that remain. As we gain more clinical experience with PFA, the benefits and limitations of the various commercial systems will become more obvious. Because of its speed and safety, PFA is likely to become the dominant mode of ablation for PVI, but we need to move forward in this field cautiously with recognition that all of the consequences of this ablation mode are still unknown.

References:

1. Verma A, Asivatham SJ, Deneke T, Castellvi Q, Neal RE. Primer on pulsed electrical field ablation: understanding the benefits and limitations. Circulation Arrhythm Electrophysiol. 2021; 14: e010086. doi.org/10.1161/CIRCEP.121.010086.

2. Napotnik TB, Polajzer T, Miklavcic D. Cell death due to electroporation - A review. Bioielectrochemistry. 2021; 141:107871.

3. Stewart MT, Haines DE, Verma A, Kirchhof N, Barka N, Grassl E, Howard B. Intracardiac pulsed field ablation: Proof of feasibility in a chronic porcine model. Heart Rhythm. 2019; 16:754-764. doi: 10.1016/j.hrthm.2018.10.030.

4. Bradley CJ, Haines DE. Pulsed field ablation for pulmonary vein isolation in the treatment of atrial fibrillation. J Cardiovasc Electrophysiol. 2020 Aug;31(8):2136-2147. doi: 10.1111/jce.14414.

5. Koruth J.S., Kuroki K., Kawamura I., et. al.: Pulsed field ablation vs radiofrequency ablation: esophageal injury in a novel porcine model. Circ Arrhythm Electrophysiol 2020; 13:

6. Neven K, van Driel VJHM, Vink A, du Pre BC, van Wessel H, Futing A, Doevendans PA, Wittkampf FHM, van Es R. Characteristics and time course of acute and chronic myocardial lesion formation after electroporation ablation in the porcine model. J Cardiovasc Electrophysiol. 2022 (in press).

7. Haines DE. Biophysics and Pathophysiology of Radiofrequency Lesion Formation. In: Catheter Ablation of Cardiac Arrhythmias, 4th Edition. Huang SKS, Miller JM, editors. Philadelphia, PA: Elsevier Saunders Publishing, 2020.

8. Nakagawa H, Castellvi, Neal R, Girouard S, Kuroda S, Hussein AA, Saliba WI, Wazni OM. Histological characterization of reversible and irreversible ventricular lesion boundaries produced by pulsed field ablation. Heart Rhythm 2021; 18: S151-S152. doi: 0.1016/j.hrthm.2021.06.386

9. Reddy VY, Neuzil P, Koruth JS, Petru J, Funosako M, Cochet H, Sediva L, Chovanec M, Dukkipati SR, Jais P. Pulsed field ablation for pulmonary vein isolation in atrial fibrillation. J Am Coll Cardiol 2019; 74: pp. 315-326. doi: 10.1016/j.jacc.2019.04.021.

10. Mukherjee RK, Roujol S, Chubb H, Harrison J, Williams S, Whitaker J, O'Neill L, Silberbauer J, Neji R, Schneider R, Pohl T, Lloyd T, O’Neill M, Razavi R. Epicardial electroanatomical mapping, radiofrequency ablation, and lesion imaging in the porcine left ventricle under real-time magnetic resonance imaging guidance-an in vivo feasibility study. Europace. 2018 Sep 1;20(FI2):f254-f262. doi: 10.1093/europace/eux341.

11. Haines DE, Wright M, Harks E, Deladi S, Fokkenrood S, Brink R, Belt H, Kolen AF, Mihajlovic N, Zuo F, Rankin D, Stoffregen W, Cockayne D, Cefalu J. Near-Field Ultrasound Imaging During Radiofrequency Catheter Ablation: Tissue Thickness and Epicardial Wall Visualization and Assessment of Radiofrequency Ablation Lesion Formation and Depth. Circ Arrhythm Electrophysiol. 2017 
Dec;10(12):e005295. doi: 10.1161/CIRCEP.117.005295.

12. Swift L, Gil DA, Jaimes R 3rd, Kay M, Mercader M, Sarvazyan N. Visualization of epicardial cryoablation lesions using endogenous tissue fluorescence. Circ Arrhythm Electrophysiol. 2014 Oct;7(5):929-37. doi: 10.1161/CIRCEP.114.001750.

Figures:

Figure 1 - A circular ablation catheter is positioned in the right superior pulmonary vein antrum (PV 1 thorugh 9) and high frequency near field pulmonary vein potentials were recorded. Immediately after delivery of a PFA pulse, the pulmonary vein potentials are eliminated.

Figure 2 - A. Irreversible electroporation waveform similar to that employed by Neven et al. from a Lifepak 9 defibrillator (Physio-control Service Manual No. 803763-06, Physio-control, Redmond, WA). B. Representative waveform from a pulsed field ablation generator using a train of biphasic microsecond duration pulses.

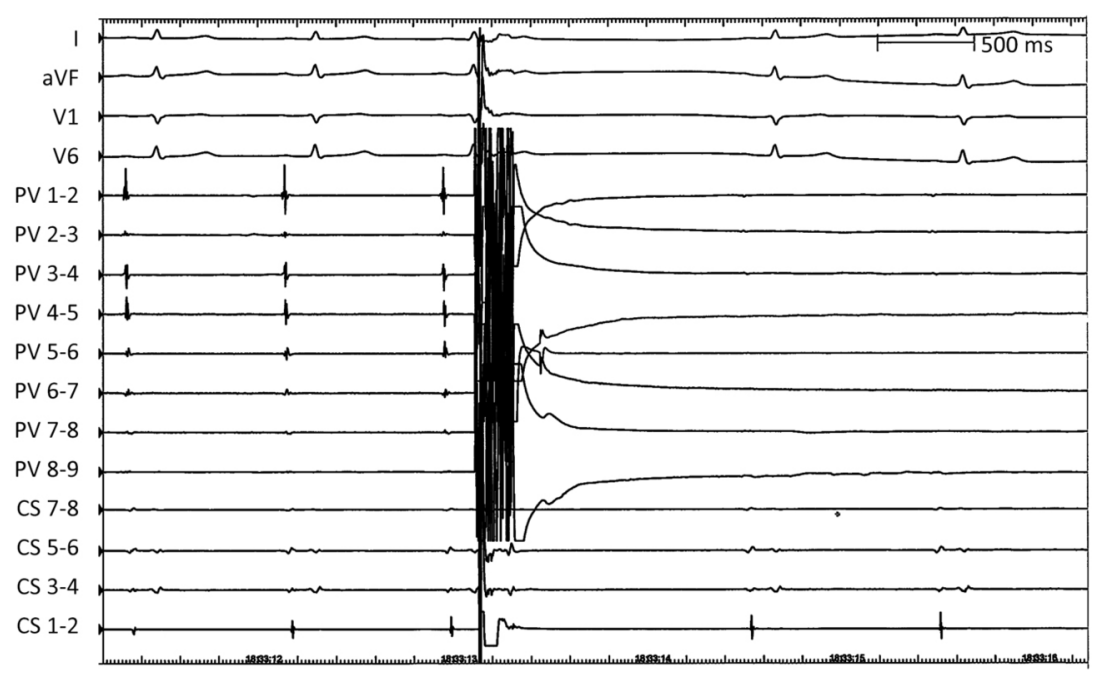

Figure 1

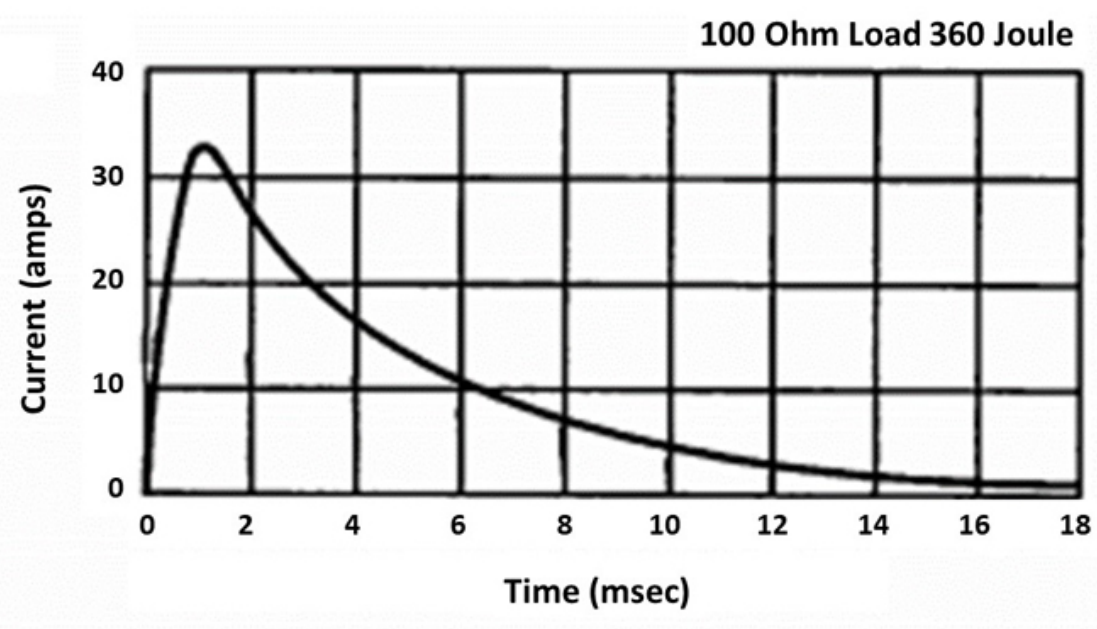


Figure 2a

Figure $2 b$
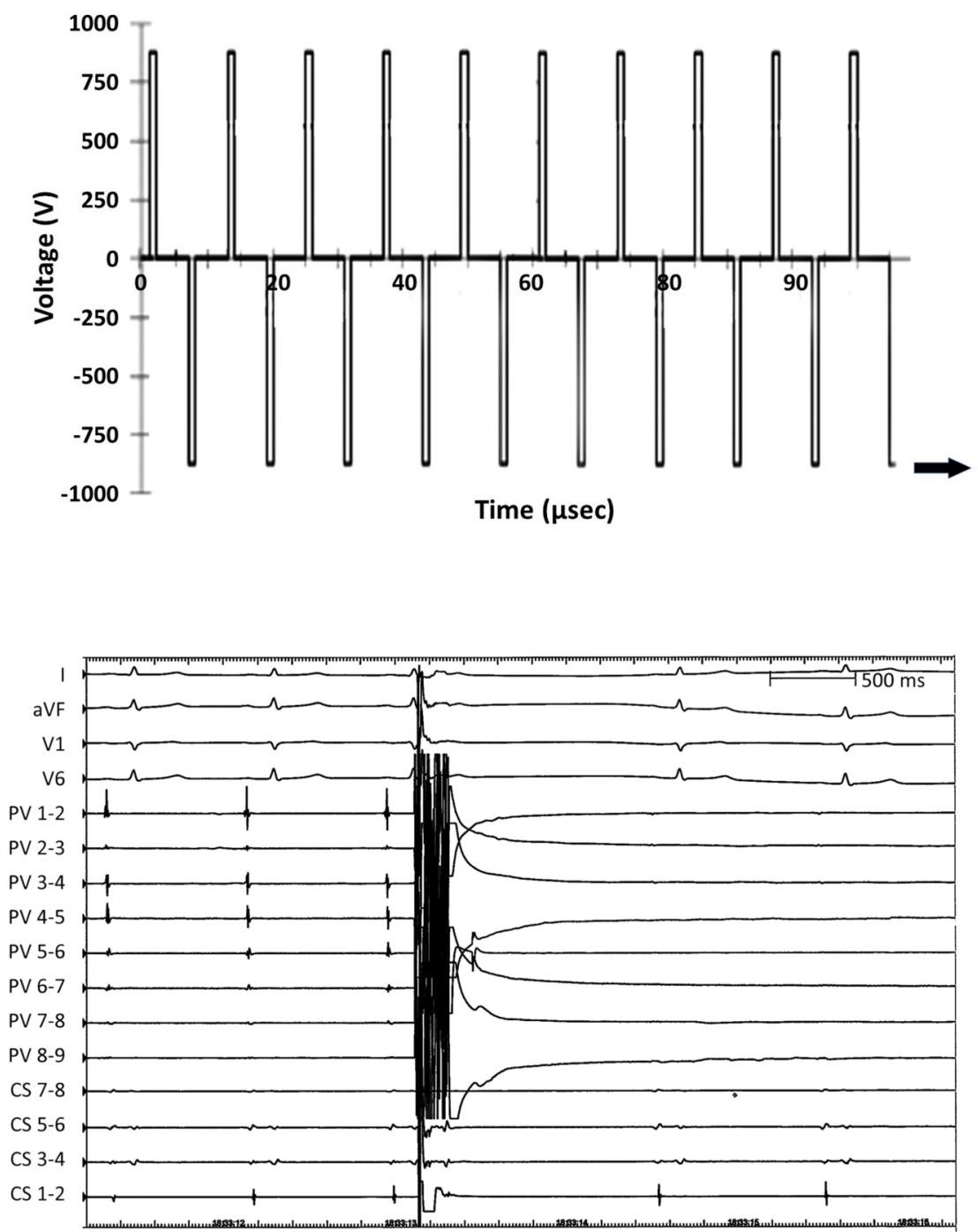
100 Ohm Load 360 Joule
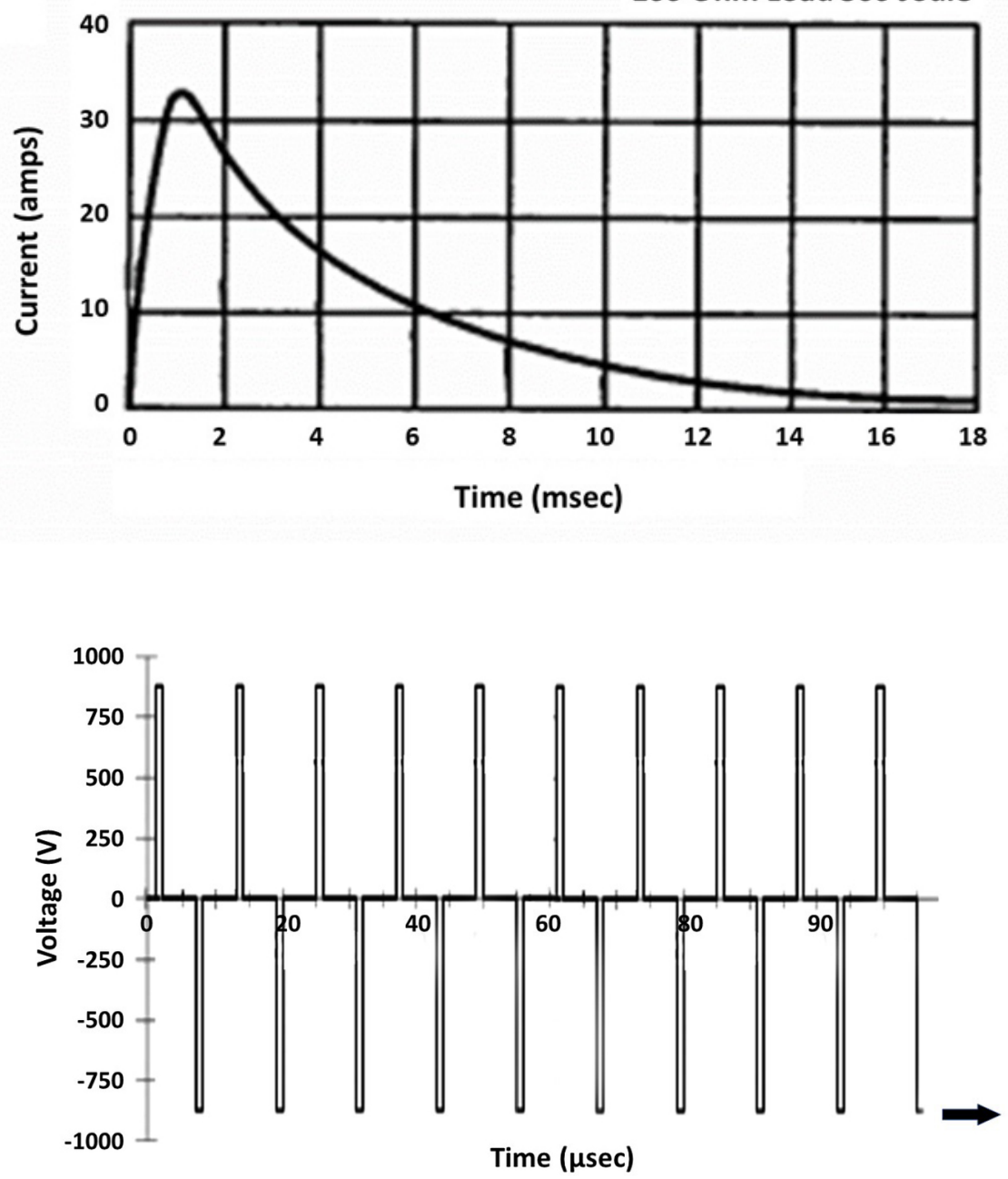